\title{
As percepções dos professores da Educação Básica sobre o uso de Tecnologias Digitais no ensino remoto emergencial
}

\author{
Sivaldo Joaquim - Universidade Federal de Alagoas (UFAL) \\ sivaldojoaquim@ic.ufal.br - https://orcid.org/0000-0002-0928-560X \\ Wilk Oliveira - Universidade de São Paulo (USP) \\ wilk.oliveira@usp.br - https://orcid.org/0000-0003-3928-6520
}

\begin{abstract}
Resumo. O distanciamento social imposto pela Covid-19 impactou a rotina educacional, levando a necessidade de ter aulas remotas com o uso de tecnologias digitais. Diante disso, analisamos a percepção de 74 professores da Educação Básica sobre o uso de tecnologias digitais no ensino remoto emergencial. Por meio de uma pesquisa qualitativa com a aplicação de um questionário misto (questões abertas e fechadas), identificou-se i) a falta de domínio, ii) a inexistência de formação para o uso de ferramentas digitais no contexto educacional, ii) o baixo nível de aproximação com os estudantes e iv) a ausência de infraestrutura para a realização das tarefas pedagógicas nas aulas remotas. $O$ estudo contribui para o desenvolvimento de políticas públicas com foco na formação inicial e continuada de professores.
\end{abstract}

Palavras-chave: Ensino Remoto, Covid-19, Tecnologias Digitais, Educação Básica, Estudo Qualitativo.

\section{The perceptions of Basic Education teachers on the use of Digital Technologies during the emergency remote education}

\begin{abstract}
The social distance imposed by Covid-19 impacted the educational routine, leading to the need to have remote classes using digital technologies. Therefore, we analyzed the perception of 74 Basic Education teachers about the use of digital technologies in emergency remote teaching. Through qualitative research with the application of a mixed questionnaire (open and closed questions), we identified i) lack of mastery of educational technologies, ii) lack of training in the use of digital tools in the educational context, ii) low level of approximation with students and iv) absence of infrastructure to carry out pedagogical tasks in remote classes. The study contributes to the development of public policies focusing on initial and continuing teacher education.
\end{abstract}

Keywords: Remote Learning, Covid-19, Digital Technologies, Basic Education, Qualitative Study.

\section{Introdução}

A educação no Brasil apresenta uma dicotomia, onde de um lado nota-se um crescimento no acesso à Educação Básica. Dado que em 2019, 98,1\% das crianças e adolescentes na faixa etária de 6 a 14 anos estavam na escola (BRASIL, 2020a, p. 24), contudo, do outro lado, observam-se os baixos índices de desempenho dos estudantes em exames nacionais e internacionais. Segundo os resultados da "Prova Brasil 2019" realizado pelo Instituto 
Nacional de Estudos e Pesquisas Educacionais Anísio Teixeira (INEP) do Ministério da Educação (MEC), apenas $57 \%$ dos estudantes aprenderam o adequado em Língua Portuguesa e $47 \%$ em Matemática, até o $5^{\circ}$ ano. Para os estudantes do $9^{\circ}$ ano, essa proporção reduz para 36\% e 18\%, respectivamente (QEDU, 2021). Além disso, o nível de proficiência dos estudantes brasileiros no Programme for International Student Assessment (PISA) em 2018 ficou abaixo da média dos países membros da Organização para a Cooperação e Desenvolvimento Econômico (OCDE) em Leitura (413 pontos), Matemática (384 pontos) e Ciências (404 pontos) comparadas às médias da OCDE: 487, 489 e 489 pontos, respectivamente (OCDE, 2019; BRASIL, 2020b).

Esses indicadores educacionais tendem a piorar com o fechamento das escolas, a evasão escolar, as dificuldades de acesso à internet e equipamentos adequados como recursos digitais para apoiar os estudantes e professores nos processos de ensino e aprendizagem durante o período de distanciamento social imposto pela pandemia do novo Coronavírus - Covid-19 (SARS-CoV-2) (Lu et al., 2020). No mundo, estima-se que no ano de 2020 aproximadamente 1,6 bilhão de estudantes e 63 milhões de professores foram impactados com o fechamento de escolas e universidades (Unesco, 2021). No Brasil, de modo especial, 5,1 milhões de estudantes na faixa etária de 6 a 17 anos não tinham acesso a Educação Básica ou estavam sem atividades escolares no final do ano letivo de 2020. Desse total, 41\% dos estudantes são crianças com idade entre 6 e 10 anos (Unicef, 2021). Ainda de acordo com o relatório do Fundo das Nações Unidas para Infância (Unicef) os maiores percentuais de estudantes fora da escola encontram-se nas regiões Norte $(28,4 \%)$ e Nordeste (18,3\%). Além disso, de acordo com a pesquisa da Unicef (2020), as crianças que residem em áreas rurais nessas regiões "são as mais atingidas pela exclusão escolar durante a pandemia em 2020" (Unicef, 2021, p, 46). Em especial, no estado de Alagoas, localizado na região Nordeste do Brasil, 17,7\% da população de 6 a 17 anos não frequentou a escola em 2020 (Unicef, 2021, p. 48).

Para minimizar os efeitos provocados pela Covid-19 na Educação Básica, muitas ações foram desenvolvidas para manter o vínculo dos estudantes com as escolas e seus professores, tais como, aulas remotas síncronas e assíncronas através de plataformas web, rádio, TV e materiais impressos (SANTOS et al., 2020; ALBUQUERQUE et al., 2021; NASCIMENTO et al., 2021). Segundo o Instituo Península (2020), “94\% dos professores indicaram que agora enxergam a tecnologia como muito ou completamente importante no processo de aprendizagem dos alunos" (INSTITUTO PENÍNSULA, 2020, p. 180). Contudo, ainda existem muitos desafios que impedem o uso efetivo das Tecnologias Digitais de Informação e Comunicação (TDIC) como ferramenta para auxiliar os professores na prática docente nos processos de ensino e aprendizagem em ambiente do mundo real. Neste cenário, uma pesquisa realizada em agosto de 2020 pelo Instituto Península (2020) mostrou que $79 \%$ dos professores relataram a falta de infraestrutura e conectividade dos alunos como o principal desafio no ensino remoto, seguido por $64 \%$ dificuldade dos professores em manter o engajamento dos alunos; $54 \%$ distanciamento e perda de vínculo com os alunos; $49 \%$ a falta de formação; $46 \%$ a falta de conhecimento em ferramentas digitais; $45 \%$ de um ambiente adequado; $38 \%$ lidar com os pais; $34 \%$ lado emocional e $26 \%$ a falta de conhecimento de recursos de acessibilidade comunicacional (INSTITUTO PENÍNSULA, 2020, p. 19).

Diante desse cenário, nesse estudo, investigamos a percepção de professores da Educação Básica a respeito da utilização das TDIC aplicada nos processos de ensino e 
aprendizagem no estado de Alagoas durante o período de distanciamento social imposto pela pandemia da Covid-19. O estudo, foi conduzido no período de agosto e setembro de 2020, com o método de pesquisa qualitativa (Creswell, 2010), com a participação de 74 professores. Os principais resultados evidenciam $i$ ) a inexistência de formação para o uso adequado das TDIC no contexto educacional durante as aulas remotas; ii) baixa aproximação dos professores com seus alunos; iii) dificuldades no uso de ferramentas digitais; $i v)$ conexão com a internet lenta; v) ausência de equipamentos e ambiente adequado para o desenvolvimento das atividades pedagógicas em home office e vi) falta de suporte técnico para auxiliar os professores. Os resultados do estudo contribuem para o desenvolvimento de políticas públicas com foco na formação inicial e continuada de professores para utilização de TDIC, especialmente aplicada na Educação Básica.

\section{Trabalhos Relacionados}

A pandemia da Covid-19 provocou mudanças radicais nos processos de ensino e aprendizagem e no cotidiano de professores e estudantes de diferentes níveis e domínios educacionais em todo o mundo (BAGATINI et al., 2020; ZUNGUZE e TSAMBE, 2020; SANTOS et al., 2020; NASCIMENTO BORBA et al., 2020; CIPRIANI et al., 2021). Dito isso, vários pesquisadores têm conduzido estudos visando analisar o impacto imposto pela Covid-19 no ambiente educacional (FREITAS VIEIRA e SILVA, 2020).

Zunguze e Tsambe (2020) conduziram um estudo com abordagens quantitativa e qualitativa com foco em analisar a percepção de 87 docentes do ensino superior de uma Universidade pública em Maputo, Moçambique, com relação ao uso de plataformas digitais como recurso tecnológico para auxiliar nos processos de ensino e aprendizagem durante o período da pandemia de Covid-19. Os resultados mostram que o E-mail $(74,7 \%)$, WhatsApp (62\%) e SIGEUP $(48,2 \%)$ um sistema de gerenciamento de aprendizagem desenvolvido pela própria Universidade foram as plataformas mais utilizadas pelos professores para disponibilização de conteúdos (Zunguze e Tsambe, 2020). Segundo os pesquisadores, leitura de textos e vídeo-aulas foram os recursos pedagógicos mais utilizados e que os professores encontraram dificuldades em gerenciar plataformas como YouTube, Zoom e Google Classroom quando comparadas a outros sistemas como WhatsApp, Facebook e ambientes institucionais (Zunguze e Tsambe, 2020).

No Brasil, Santos et al. (2020), realizaram um estudo de caso com abordagem de pesquisa qualitativa envolvendo 36 professores da Educação Básica de uma escola pública municipal, visando identificar as possibilidades, dificuldades e percepções com relação à utilização da plataforma Google Classroom. Os principais resultados revelaram que houve dificuldades na implementação da plataforma, tais como: falta de conectividade com a Internet, capacitação dos docentes e orientações para os estudantes utilizarem o sistema de forma adequada (Santos et al., 2020). Dificuldades semelhantes foram apontadas no estudo de Zunguze e Tsambe (2020).

Nascimento Borba et al. (2020), realizaram uma pesquisa com 187 docentes para compreender as práticas pedagógicas de ensino de Ciências e Biologia no contexto da Educação Básica durante o período de isolamento social imposto pela pandemia da Covid-19 no Brasil. Os principais resultados mostram dificuldades relacionadas a conectividade de professores e estudantes; ambiente doméstico (home office) inapropriado; sobrecarga de trabalho; falta de domínio para o uso de tecnologias digitais; 
dúvidas sobre metodologias para o ensino online e problemas relacionados à saúde. Durante esse período, os professores produziram e/ou utilizaram listas de exercícios, textos científicos, reportagens, ambientes virtuais de aprendizagem (Moodle e Google Classroom), vídeos e documentários (YouTube e outros sites), aulas online ao vivo (videoconferência), aulas gravadas por outros professores, simulador $(P h E T)$ e jogos/ambientes gamificados (Kahoot) como recursos pedagógicos nos processos de ensino e aprendizagem.

Ainda mais recente, Cipriani et al. (2021) conduziram um estudo com análise de conteúdo e estatística descritiva com 209 professores da Educação Básica visando analisar os pensamentos, sentimentos, desafios e perspectivas dos docentes durante o período de distanciamento social. Os resultados mostram que as tecnologias digitais mais utilizadas foram: Google Meet (22,58\%), Microsoft Teams (21,89\%), YouTube (12,67\%), Google Classroom (11,98\%), Hangouts (8,53\%), Zoom (7,37\%), WhatsApp (2,76), a plataforma Moodle (2,30\%), outros recursos digitais $(9,22 \%)$ e nenhuma tecnologia (0,69\%) (CIPRIANI et al., 2021).

Em resumo, os trabalhos relacionados mostram que houve uma sobrecarga do trabalho docente na modalidade de ensino remota e os profissionais tem relatados preocupações, ansiedade, falta de feedback, entre outros problemas com a utilização das TDIC para auxiliar nos processos de ensino e aprendizagem em diferentes níveis educacionais.

\section{Materiais e Método}

O presente estudo tem como objetivo analisar a percepção de professores da Educação Básica do estado de Alagoas sobre a utilização das TDIC em diferentes domínios do conhecimento e níveis educacionais no contexto do ensino remoto emergencial (causado pela pandemia da Covid-19).

Para isso, foi utilizado como método de pesquisa uma abordagem qualitativa com ênfase na aplicação de um survey online desenvolvido na plataforma Google Forms com 32 questões objetivas e 11 subjetivas, totalizando 43 questões divididas em quatro seções: i) Termo de Consentimento Livre e Esclarecido - TCLE; ii) questões demográficas; iii) sobre o distanciamento social; e (iv) sobre o uso de tecnologias no ensino remoto emergencial.

Na primeira seção, apresentamos o título da pesquisa, o objetivo principal e o TCLE com informações claras para os participantes e contato do pesquisador responsável para sanar possíveis dúvidas. Na segunda seção, os participantes foram convidados a responder 12 questões sobre o seu perfil e atuação profissional. Na terceira seção, foram apresentadas cinco questões relacionadas ao distanciamento social. Por último, na quarta seção, os participantes foram convidados a responder 26 questões sobre o uso de tecnologias no período da pandemia da Covid-19.

No questionário, foi incluída uma questão para "checar atenção" (i.e., essa é uma questão apenas para entender se você está lendo as questões com a devida atenção. Se você leu essa questão, por favor, marque a opção “4”), visando eliminar respostas em que os participantes não tiveram a devida atenção ao responder a pesquisa. 


\subsection{Coleta e análises dos dados}

A coleta de dados ocorreu nos meses de agosto e setembro de 2020 através do questionário online enviado por meio de grupos de professores e coordenações pedagógicas da Educação Básica no WhatsApp. Após isso, foi realizado análise dos dados. Nesta etapa constatamos que 78 participantes responderam à pesquisa.

No escopo deste estudo, foram utilizadas apenas as questões fechadas sobre o perfil dos participantes e as questões abertas. Além disso, foram aplicados os seguintes critérios de inclusão: $i$ ) ser professor atuante da Educação Infantil, Ensino Fundamental I e II, Ensino Médio, Educação de Jovens e Adultos (EJA) e ii) ofertar aulas remotas na instituição de ensino onde o participante leciona durante a pandemia da Covid-19. Como critérios de exclusão: i) não aceitar o TCLE e ii) não marcar a opção 4 da questão "checagem de atenção" para entender se estava lendo atentamente.

Após aplicar os critérios de inclusão e exclusão, foi constatado que 4 participantes não estavam atuando como professor durante a pandemia. Os dados destes participantes foram removidos da base de dados. Dessa forma, o número total de participantes considerado neste estudo foi de 74 professores da Educação Básica.

\subsection{Participantes}

Os participantes são 74 professores da Educação Básica que lecionam no estado de Alagoas na faixa etária de 23 a 62 anos de idade $(\bar{X}=40,49, M d=40$ e $S=7,84)$, com experiência docente entre 1 e 40 anos $(\bar{X}=14,26, M d=14$ e $S=8,14)$. Desse total, 53 $(71,62 \%)$ são do sexo feminino e $21(28,38 \%)$ masculino. Sendo que $42(56,76 \%)$ desses profissionais atuam em escolas da rede pública de ensino (municipal e estadual), 25 $(33,78 \%)$ na rede de ensino particular e $7(9,46 \%)$ atuam em ambas as instituições de ensino (pública e particular). Com relação à formação dos professores, 24 (32,43\%) possuem graduação, $47(63,51 \%)$ especialização e $3(4,06 \%)$ mestrado. Esses profissionais atuam em diferentes etapas da educação básica, conforme mostrado na Tabela 1.

Tabela 1. Etapas de atuação dos professores da Educação Básica

\begin{tabular}{l|c|r}
\hline Etapas da Educação Básica & Quantidade de Participantes & Percentual (\%) \\
\hline EI & 08 & 10,81 \\
\hline EI e EF I & 02 & 2,70 \\
\hline EF I & 15 & 20,27 \\
\hline EF I e EF II & 02 & 2,70 \\
\hline EF II & 19 & 25,68 \\
\hline EF II e EJA & 01 & 1,35 \\
\hline EJA & 01 & 1,35 \\
\hline EF II e EM & 13 & 17,57 \\
\hline EM & 13 & 17,57 \\
\hline Total de participantes & $\mathbf{7 4}$ & $\mathbf{1 0 0 . 0 0}$ \\
\hline Legenda: EI - Educação Infantil; EF I - Ensino Fundamental I; EF II - Ensino \\
Fundamental II; EJA - Educação de Jovens e Adultos; EM - Ensino Médio. \\
\hline
\end{tabular}

\section{Resultados e Discussão}

Nesta seção, são apresentados os resultados extraídos com base na análise qualitativa envolvendo os 74 professores participantes da pesquisa, bem como, as discussões acerca dos resultados. Os primeiros questionamentos foram: como era sua relação com os seus 
alunos antes da pandemia (em termos de conversação/diálogos)?; Como tem sido sua relação com os seus alunos durante a pandemia (em termos de conversação/diálogos)?

Conforme pode ser observado na Tabela 2, antes da pandemia nenhum dos professores afirmaram que a relação era muito distante, enquanto que, durante a pandemia observou-se um aumento de 4,1\%. Além disso, notou-se que 4,1\% disseram sentir-se distante dos estudantes antes da pandemia, esse percentual aumentou para 35,1\% durante a pandemia. Antes, 52,7\% afirmaram manter uma relação próxima com os estudantes, já durante a pandemia essa aproximação foi de apenas 44,6\%. Quando questionados sobre estar muito próximo dos estudantes, $43,2 \%$ disseram que sim, antes da pandemia. Durante o distanciamento social, esse percentual foi de apenas $16,2 \%$, conforme mostrado na Tabela 2.

Tabela 2. Relação dos professores com seus alunos antes e durante o período de distanciamento social imposto pela Covid-19

\begin{tabular}{l|c|c}
\hline Nível de aproximação & Antes & Durante \\
\hline Muito distante & $0,0 \%$ & $4,1 \%$ \\
\hline Distante & $4,1 \%$ & $35,1 \%$ \\
\hline Próxima & $52,7 \%$ & $44,6 \%$ \\
\hline Muito próxima & $43,2 \%$ & $16,2 \%$ \\
\hline
\end{tabular}

Em relação a sua prática docente, quais as dificuldades para uso das tecnologias digitais e/ou metodologias de ensino e aprendizagem em ambiente online? As principais dificuldades relatadas pelos professores participantes foram à inexistência de formação/capacitação para o uso adequado das tecnologias digitais no cenário educacional, de plataformas como Google Classroom e Meet, a falta de domínio para editar vídeos, fotos, podcasts e para apresentar vídeos durante as reuniões remotas.

Relatos semelhantes foram apresentados por Santos et al. (2020) ao analisar as possibilidades e dificuldades do uso do Google Sala de Aula em uma escola pública no município de Torres, Rio Grande do Sul. Neste contexto, um professor "A", relatou ter dificuldades "na utilização de aplicativos matemáticos" nos ambientes de ensino online, outros professores destacaram pontos relacionados à falta de treinamento, o professor "B" disse que precisa "ter mais habilidades nas ferramentas de videoconferência e ambientes virtuais de aprendizagem", o professor "C" afirmou ter "dificuldade para assimilação rápida dessas tecnologias. Preciso de tempo e prática para aprender com eficácia”. No geral, nota-se uma necessidade urgente de capacitação docente (Nobre \& Rodrigues, 2020) para aplicação das TDIC no contexto educacional.

Relatos semelhantes foram apontados por outros professores: "é difícil verificar a qualidade do ensino de modo remoto tendo em vista alguns empecilhos que impedem a efetivação do ensino. Muitos pesquisam na internet, fazem a atividade sem muita motivação e empenho"; "a falta de prática de meter em áreas ainda desconhecidas, não ter o contato direto com os alunos, a internet cair demais e a falta de treinamento pra essa área"; "não conhecer direito as funções de alguns apps e timidez"; "não consigo aprender com facilidade nada. Minha filha que me ajuda"; "é dificil falar para uma câmera, poucos estudantes interagem".

Um professor comentou em "diversificar o uso dessas ferramentas" com a utilização de jogos digitais (Joaquim \& Oliveira, 2020; Oliveira \& Joaquim, 2020) para aumentar o engajamento. Desafios como esses foram identificados em uma pesquisa realizada pelo Instituto Península com professores da Educação Básica no Brasil. Segundo a pesquisa, " $88 \%$ dos professores afirmaram no início da pandemia que nunca 
tinham dado aula de forma virtual antes" (INSTITUTO PENÍNSULA, 2020, p. 8). No Brasil, a falta de formação adequada para o uso pedagógico das TDIC no contexto educacional não é restrita apenas aos professores da Educação Básica, Araujo et al. (2020) afirmam que no Ensino Superior, nem todos os professores "estavam preparados para esta mudança tão repentina" (ARAUJO et al., 2020, p. 1).

Além das dificuldades relacionadas o uso das TDIC, o presente estudo buscou entender se o distanciamento social prejudicou de alguma forma o trabalho como professor? Se sim, de que maneira?, $26(35,1 \%)$ responderam que não e $48(64,9 \%)$ professores disseram que sim, prejudicou o trabalho docente. Um professor expressou: "tenho dedicado muito mais tempo a edição de materiais de apoio para as aulas do que o habitual devido pandemia, o que tem tornado um período muito maior frente ao celular e ao computador".

Vários estudos (ARAUJO et al., 2020; CIPRIANI et al., (2021); FREITAS VIEIRA e SILVA, 2020; NASCIMENTO BORBA et al., 2020; INSTITUTO PENÍNSULA, 2020) apontam uma sobrecarga das atividades docentes no período da pandemia da Covid-19. Para outro professor "as aulas tornaram-se mecânicas, com pouca ou nenhuma interação". A ausência de feedback e engajamento dos estudantes têm sido uma das preocupações dos professores no contexto da pandemia da Covid-19. Diante o exposto, Tavares et al. (2020) propõem o uso de ferramentas como a rede social "Instagram" para ampliar a interação entre professores e estudantes nos processos de ensino e aprendizagem no domínio de Língua Portuguesa. No entanto, a falta de equipamentos adequados e conexão com a internet para os estudantes participarem das aulas online é um problema comum nos municípios no interior do Nordeste brasileiro.

Segundo um professor "a maioria dos alunos não acompanham as atividades desenvolvidas por falta de recursos materiais e culturais". E isso contribui para ausência nas aulas e consequentemente o agravamento dos baixos índices de desempenho de aprendizagem dos estudantes da Educação Básica (QEDU, 2021; OCDE, 2019). Outro professor relata que sente falta "do contato direto com os alunos". Relatos dessa natureza tem chamado a atenção dos professores da área de exatas, um docente destaca que sente dificuldades "para saber se aprenderam os conteúdos". Além disso, questionamos aos professores se as TDIC têm ajudado a superar as dificuldades? Se sim, de que maneira? Dos 74 professores, $27 \%$ disseram que as TDIC não ajudaram e $73 \%$ afirmaram que sim. Para esses professores, as TDIC auxiliaram de diferentes maneiras. De acordo com um professor: "tem me aproximado dos alunos, além de não sofrer tantos impactos negativos neste distanciamento social". Outros docentes afirmaram que: "a partir delas e principalmente do WhatsApp é possível as aulas remotas", "torna as aulas mais atrativa", "melhores didáticas de ensino", e contribuiu para a "comunicação virtual".

Esses resultados possibilitaram responder a principal questão de pesquisa: como as TDIC têm impactado nas práticas pedagógicas dos professores da Educação Básica durante o período de distanciamento social imposto pela pandemia da Covid-19? De modo geral, os professores perceberam o potencial das TDIC nos processos de ensino e aprendizagem. Sendo assim, apesar das limitações de equipamentos e conexão com internet, um docente expressou: "sem as TDIC não poderíamos dar continuidade ao ano letivo de 2020". A percepção dos professores é de que as TDIC impactaram de forma positiva no diálogo entre os atores do sistema educacional (estudantes, professores, coordenadores, diretores e famílias) por meio de ferramentas digitais (e.g., Google Meet 
e WhatsApp). Além disso, permitiu o desenvolvimento de atividades pedagógicas de forma síncrona e assíncrona com o uso de plataformas web (e.g., Google Classroom, Meet e redes sociais), que possibilitou com maior agilidade o compartilhamento de materiais didáticos-pedagógico (textos, imagens, gráficos, vídeos, formulários, entre outros) através do Google Drive.

Por outro lado, o cenário sobrecarregou os docentes no desenvolvimento das atividades pedagógicas em home office no período de distanciamento social. Mas também despertou o interesse em buscar novos conhecimentos para aplicação das tecnologias educacionais digitais em diferentes domínios e níveis educacionais. Além disso, possibilitou a aquisição de competências e habilidades da cultura digital (Brasil, 2018) essenciais para o desenvolvimento da Educação Básica.

\subsection{Limitações e recomendações para ações futuras}

O estudo apresentado nesse artigo possui algumas limitações que fazem parte da própria natureza do estudo. Inicialmente, os resultados podem ser representativos apenas na região onde o estudo foi conduzido. Apesar de essa ter sido uma decisão de projeto com o objetivo de entender como os professores do interior do Brasil estavam lidando com a necessidade de ministrar aulas remotas por meio de sistemas educacionais online, sugerimos que estudos futuros repliquem nossa pesquisa em outras regiões do país. Ao mesmo tempo, por se tratar de um estudo qualitativo realizado de maneira remota, algumas informações podem não terem sido devidamente abordadas nas análises. Por isso, recomendamos que estudos futuros também possam realizar análises quantitativas.

Com base nos resultados obtidos nesse estudo, é possível aprender algumas lições importantes as quais geram recomendações para ações futuras, tais como: i) o desenvolvimento de políticas públicas voltadas para a criação, inserção e utilização de forma efetiva das TDIC na Educação; ii) investimentos públicos na formação inicial e continuada de professores da Educação Básica para utilização das TDIC de forma adequada visando aumentar a motivação, engajamentos e consequentemente melhorar o desempenho de aprendizagem dos estudantes; iii) investimentos em infraestrutura tecnológica no ambiente escolar; $i v)$ aquisições e disponibilização de recursos digitais (e.g., tablets, smartphones, notebooks, softwares e plataformas educacionais) para professores e estudantes e $v$ ) investigações diagnósticas periódicas para monitorar, analisar e compreender a realidade de estudantes, professores e instituições de ensino com o uso de tecnologias educacionais.

\section{Considerações Finais}

Os principais resultados desse estudo mostram um distanciamento dos professores na relação com os estudantes durante o período da pandemia da Covid-19. Isso é preocupante porque pode contribuir para o aumento da evasão escolar e impacta no baixo desempenho de aprendizagem dos estudantes da Educação Básica (QEDU, 2021; OCDE, 2019). Outro ponto observado foi a ausência de formação dos docentes para o uso adequado TDIC no contexto educacional. Além disso, a falta de suporte técnico, infraestrutura, conexão lenta com a internet e recursos digitais apropriados para utilização pelos professores e estudantes durante esse período contribuíram para a baixa aproximação dos professores com seus estudantes nos processos de ensino e aprendizagem. Como trabalhos futuros, pretende-se realizar um estudo com abordagem de pesquisa mista (qualitativa e 
quantitativa) sobre o impacto das TDIC nas práticas pedagógicas dos professores da Educação Básica em diferentes regiões do Brasil.

\section{Referências}

Albuquerque, L. C., Diniz, É. L., Araújo, E. J. M., \& Baptista, M. D. G. D. A. (2021). Percepções discentes sobre aulas remotas em tempos de pandemia. Debates em Educação, 13(31), 927-942.

Araujo, R. M., Cibelle, A., Martins, V. F., Eliseo, M. A., \& Silveira, I. F. (2020). COVID19, Mudanças em Práticas Educacionais e a Percepção de Estresse por Docentes do Ensino Superior no Brasil. Revista Brasileira de Informática na Educação, 28, 864891.

Bagatini, D., Soares, R., Barros, E., \& Silveira, G. (2020). Estudo virtual em tempos de distanciamento: uma análise sobre o impacto do isolamento social na rotina de estudos de universitários. In Anais do XXXI Simpósio Brasileiro de Informática na Educação, (pp. 792-801). Porto Alegre: SBC. doi:10.5753/cbie.sbie.2020.792

Brasil, Instituto Nacional de Estudos e Pesquisas Educacionais Anísio Teixeira (Inep). (2020a). Relatório do $3^{\circ}$ ciclo de monitoramento das metas do Plano Nacional de Educação - 2020: sumário executivo. Brasília.

Brasil, Instituto Nacional de Estudos e Pesquisas Educacionais Anísio Teixeira. (2020b). Relatório Brasil no PISA 2018. Brasília: Inep/MEC.

Brasil, Ministério da Educação. (2018). Base Nacional Comum Curricular. Disponível em: $<$ http://basenacionalcomum.mec.gov.br/abase $>$ Acesso em: 25 jul. 2021.

Cipriani, F. M., Moreira, A. F. B., \& Carius, A. C. (2021). Atuação Docente na Educação Básica em Tempo de Pandemia. Educação \& Realidade, 46.

Creswell, J. W. (2010). Projeto de Pesquisa: métodos qualitativo, quantitativo e misto. $3^{\text {a }}$ ed. - Porto Alegre: Artmed.

Freitas Vieira, M., \& da Silva, C. M. S. (2020). A Educação no contexto da pandemia de COVID-19: uma revisão sistemática de literatura. Revista Brasileira de Informática na Educação, 28, 1013-1031.

Instituto Península. (2020). Sentimento e percepção dos professores brasileiros nos diferentes estágios do Coronavírus no Brasil.

Joaquim, S., \& Oliveira, W. (2020). Ensinando Operações Matemáticas com o Uso de Jogos Digitais no Ensino Fundamental. In Anais do XXVI Workshop de Informática na Escola, (pp. 71-80). Porto Alegre: SBC. doi:10.5753/cbie.wie.2020.71.

Lu, X., Zhang, L., Du, H., Zhang, J., Li, Y. Y., Qu, J., ... \& Wong, G. W. (2020). SARSCoV-2 infection in children. New England Journal of Medicine, 382(17), 1663-1665.

Nascimento, I. M., do Amaral Neto, J. R., da Silva Junior, L. O., de Lima Costa, T. K., \& Oliveira, W. (2021). Tentando Recuperar a Interação Social Perdida Durante a Pandemia da Covid-19 por meio da Gamificação. In Anais do XXVII Workshop de Informática na Escola (pp. 212-223). SBC.

Nascimento Borba, R. C., Teixeira, P. P., Fernandes, K. D. O. B., Bertagna, M., Valença, C. R., \& de Souza, L. H. P. (2020). Percepções docentes e práticas de ensino de 
ciências e biologia na pandemia: uma investigação da Regional 2 da SBEnBio. Revista de Ensino de Biologia da SBEnBio, 153-171.

Nobre, A., \& Rodrigues, C. (2020). Experiências da Formação do Docente do Ensino Superior no Google Classroom em Tempos da Pandemia da COVID-19. In Anais do XXVI Workshop de Informática na Escola, (pp. 339-348). Porto Alegre: SBC. doi:10.5753/cbie.wie.2020.339.

OECD, PISA 2018. (2019). Results: Excellence and Equity in Education. Volume I. Paris: OECD Publishing.

Oliveira, W., \& Joaquim, S. (2020). A Influência dos Jogos Educativos Analógicos e Digitais na Interação Social dos Estudantes. In Anais do XXVI Workshop de Informática na Escola, (pp. 409-418). Porto Alegre: SBC. doi:10.5753/cbie.wie.2020.409.

Qedu, Portal QEdu. (2021). Disponível em: <https://qedu.org.br/brasil/aprendizado>. Acesso em: 05 jul. 2021.

Santos, C., Paillard, G., Moreira, L., Silva Filho, F., \& Coutinho, E. (2020). Uma Análise Qualitativa sobre Atividades Remotas em Disciplinas no Período de Isolamento Social. In Anais do XXXI Simpósio Brasileiro de Informática na Educação, (pp. 292301). Porto Alegre: SBC. doi:10.5753/cbie.sbie.2020.292

Santos, M., de Sousa Júnior, A. R., Machado, L. R., \& Bilessimo, S. M. S. (2020). Possibilidades e dificuldades na utilização do Google Sala de Aula: um estudo de caso em uma escola pública Brasileira. RENOTE, 18(2), 49-58.

Tavares, A., Sales, E., Ramos, E., Santos Júnior, I., Oliveira, I., Silva, M., Andrade, P., $\&$ Medeiros, S. (2020). Construção de um Guia para o Uso do Instagram no Contexto do Ensino Remoto: Empatia Durante a Pandemia da Covid-19. In Anais do XXVI Workshop de Informática na Escola, (pp. 289-298). Porto Alegre: SBC. doi:10.5753/cbie.wie.2020.289.

Unesco, Organização das Nações Unidas para a Educação, a Ciência e a Cultura. (2021). COVID-19 impact on education. Disponível em: $<$ https://en.unesco.org/covid19/educationresponse>. Acesso em: 28 jun. 2021.

Unicef, Fundo das Nações Unidas para a Infância. (2021). Cenário da Exclusão Escolar no Brasil: Um alerta sobre os impactos da pandemia da COVID-19 na Educação. Disponível em: $<$ https://www.unicef.org/brazil/relatorios/cenario-da-exclusaoescolar-no-brasil>. Acesso em: 28 jun. 2021.

Zunguze, M. C., \& Tsambe, M. Z. A. (2020). Perception of Teachers in the use of Electronic Platforms to Support Face-to-Face Teaching During the term of The State of Emergency due to Covid-19: Case of the Pedagogical University of Maputo. RENOTE, 18(2), 40-48. 